\title{
POSGRADOS UNIVERSITARIOS: ¿ACTIVIDAD ACADÉMICA O SERVICIO AL CLIENTE? EL CASO DE LA UNIVERSIDAD DE BUENOS AIRES
}

Marcela Mollis*

Guillermo Jaim Etcheverry** 


\section{RESUMEN}

El artículo entrega información cuantitativa sobre los posgrados de la Universidad de Buenos Aires, en el marco de la oferta de posgrados a nivel nacional y regional.

Analiza los diferentes sectores institucionales -especialización, maestría y doctorado- desde la perspectiva del desempeño académico y el cumplimiento de su misión específica.

Por último, plantea una reflexión sobre las urgencias pendientes para promover posgrados de excelencia que respondan a las necesidades cívicas, a la formación cultural, científica y tecnológica, en el contexto de la regionalización y mundialización económica.

\section{ABSTRACT}

The paper provides quantitative data on graduate degrees offered by the Universidad de Buenos Aires, in the context of the national and regional supply of graduate courses.

It analyzes the different institutional sectors-specialization, master's degrees and doctorates- from the point of view of academic performance and the fulfillment of their specific mission.

Lastly, it reflects on urgent matters that are still outstanding in order to promote graduate degrees at a level of excellence which will answer to the needs of civil society, to cultural training in science and technology in the context of economic globalization and regionalization. 


\section{POSGRADOS UNIVERSITARIOS: ¿ACTIVIDAD ACADÉMICA O SERVICIO AL CLIENTE? EL CASO DE LA UNIVERSIDAD DE BUENOS AIRES}

\section{CONTEXTO INTERNACIONAL DE LOS POSGRADOS: REGIONALIZACIÓN Y MUNDIALIZACIÓN}

En las últimas décadas, la formación profesional de alto nivel se ha realizado a través de programas de posgrado que se han expandido en todo el mundo. En los países que concentran la riqueza, los posgrados de mayor calidad se vinculan directamente a la capacidad de producción de conocimiento científico-tecnológico, como lo señalan algunos documentos del Banco Mundial, el Banco Interamericano de Desarrollo (BID) y la Organización para la Cooperación y el Desarrollo Económico (OECD).

Paradójicamente en América Latina, la explosión de los posgrados -que no pocas veces se asocia a un debilitamiento evidente de la enseñanza de grado- ha carecido del impacto esperado respecto a la producción de conocimiento innovador y la capacitación de quienes se dedican a la ciencia y la tecnología. Algunos autores coinciden en que el personal ha optado por desarrollar proyectos individuales, ligados en general a actividades de consultoría internacional, regional e incluso corporativa, y han respondido, también, a las demandas de la comunidad científica internacional. De este modo, se ha producido el fenómeno de la pérdida de cerebros (brain loss) en aquellos casos que se vincularon a proyectos diferentes al área para la que fueron formados o la fuga de cerebros (brain drain) cuando abandonaron el país que financió su formación (RICYT, 2000) ${ }^{1}$.

$\overline{1}$ El informe de la RICYT del año 2000 presenta indicadores que permiten cuantificar el problema de la escasez de recursos destinados a las actividades científicas y tecnológicas en la región, tanto en lo que se refiere a la dotación de científicos e ingenieros como al financiamiento. Al mismo tiempo, se diferencian trayectorias nacionales muy disímiles al respecto. Brasil y Chile han puesto en marcha proyectos para elevar la inversión en Ciencia y Tecnología, en cambio, Argentina muestra una inversión inferior al uno por ciento del PBI, con tendencia al estancamiento. 
En la mayoría de los posgrados de América Latina se genera una tensión entre las actividades de formación en investigación y el entrenamiento profesional en áreas especializadas. Esta falsa oposición da por resultado la explosión tanto en la relevancia institucional como en la matrícula de estos últimos. Entre los factores que contribuyen al desarrollo del mercado de los posgrados profesionales, es posible señalar:

a) la carencia de una política científica sostenida por parte de los estados nacionales de la región,

b) la ausencia de un sector empresario que demande resultados del campo científico para aplicarlos a la producción, y

c) un contexto internacional en el que las grandes corporaciones importan no solo el saber sino, además, la tecnología y el personal jerárquico. Sin embargo, resulta importante señalar que una parte considerable de la investigación científica y tecnológica en la región se realiza de modo preponderante en el sector público, en las universidades y, especialmente, en el nivel de los doctorados ${ }^{2}$.

Los posgrados que se originaron en la década de 1950, nucleados en torno a las facultades de medicina, aumentaron lentamente en la década siguiente y se afirmaron durante los años 1979 y 1980. Incluso, en la difícil década de 1980 se duplicó el promedio de la matrícula considerada en toda la región. Los 74.000 estudiantes de comienzos de la década aumentaron a cerca de 150.000 alumnos, a fines de la misma (García Guadilla, C. 1994).

La estructura de los posgrados desarrollada en las dos últimas décadas se ha expandido y consolidado, especialmente en países como Brasil, México, Venezuela, Colombia y Chile, mediante la construcción de una capacidad institucional endógena. Carmen García Guadilla describe una tipología en los inicios de los años 1990 que permite distinguir grandes diferencias entre los distintos países de América Latina. De acuerdo con el volumen de la matrícula, resulta posible identificar cuatro grupos de países:

2. Carmen García Guadilla (1996) señala que América Latina cuenta con más de 180 mil estudiantes de posgrado. De ellos, el 75 por ciento pertenece al sector público y en el caso de los doctorados, cerca del noventa por ciento se encuentra en las universidades públicas. 
1) aquellos como Brasil y México, que cuentan con una población de más de cincuenta mil estudiantes de posgrado;

2) Venezuela, Perú, Colombia, Chile y Argentina, con una población de entre seis mil y doce mil;

3) Bolivia, Cuba, Costa Rica y Uruguay, con una población de entre quinientos y $2.300 \mathrm{y}$

4) el resto de los países, cuya población de posgrado es de menos de quinientos estudiantes.

La explosión de posgrados de la década de 1990 trajo aparejada la necesidad de evaluarlos y acreditarlos. Con tal finalidad, se estructuraron organismos locales de evaluación y acreditación, con funciones organizacionales semejantes, como lo evidencian las ponencias presentadas por los investigadores participantes en el Taller Internacional "Evaluación y Acreditación de la Educación Superior en América Latina y el Caribe"3.

\section{EXCELENCIA, EVALUACIÓN Y ACREDITACIÓN DE LOS POSGRADOS EN ARGENTINA}

Se puede definir excelencia como una dimensión de la calidad asociada con el máximo logro referido a los parámetros intrínsecos al desarrollo de cualquier disciplina. En cambio, valor comprende una dimensión utilitaria de la calidad, cuya expresión más frecuente, aunque no única, está vinculada con el mercado. Así lo expresa Christopher Ball:

Se puede decir que aquel estudiante que estudió Ingeniería y se recibió con una calificación promedio es valioso para la industria pero no logró la excelencia en sus estudios. Por el contrario, se podría afirmar que el estudiante que hizo la carrera de letras inglesas y recibió los más altos honores académicos se desempeñó con la excelencia en la universidad pero es menos valioso para la sociedad.

(Ball 1991:65, citado en Mollis 1996)

3. Evento auspiciado por la CONEAU y el IESALC, realizado en la ciudad de Buenos Aires entre el 8 y 9 de mayo de 2003. Consultas del material digitalizado o la versión electrónica, en la siguiente dirección IESALC/UNESCO, boletín@unesco.org.ve 
Estas consideraciones obligan a introducir en un sistema de evaluación de la calidad de la educación superior tanto parámetros extrínsecos como intrínsecos al trabajo académico, si se aspira evaluar excelencia y valor de manera conjunta.

El principal riesgo que supone asimilar calidad y valor, excluyéndose la dimensión de la excelencia en la construcción de los sistemas de evaluación de la calidad, reside en medir alguna función específica, cuyo logro no formó parte del proyecto institucional que fundó y refundó cada universidad en su trayectoria histórica. En otras palabras, lo que la sociedad-mercado define como valioso en un momento dado, no refleja el proyecto institucional por el cual fue creada la universidad y que, a su vez, actúa como mandato histórico o como la leyenda organizacional (Brünner, J. J 1990) que da sentido e identidad a la propia institución.

En Argentina, entre 1996 y 2001, se acreditaron 936 posgrados, de los cuales 441 son especializaciones, 332 maestrías y 163 doctorados. A su vez, el número más importante de especializaciones acreditadas pertenece a Medicina, que representa el 54 por ciento del total. Le siguen las especializaciones en Ciencias Jurídicas, con el 10,4 por ciento y en Administración, con un 7,3 por ciento del total.

En cuanto a las maestrías, los porcentajes más altos se reparten entre Administración, Agronomía e Ingeniería con un 9,9 por ciento, 9,6 por ciento y 11,4 por ciento, respectivamente. La situación de los doctorados muestra que el mayor porcentaje de programas acreditados se distribuye entre Ingeniería con un quince por ciento, y le siguen Ciencias Biológicas, Ciencias Jurídicas, Física y Química con cerca de un siete por ciento cada una ${ }^{4}$.

Las especializaciones y maestrías que se han acreditado hasta ahora en la Argentina no representan necesariamente los posgrados de excelencia, aunque sí reproducen los parámetros de calidad asociados con el valor que el grupo de pares evaluadores y la Comisión Nacional de Evaluación y Acreditación Universitaria (CONEAU) asignaron a cada posgrado, en el momento y contexto en el que la acreditación fue realizada.

4. Estos datos fueron elaborados a partir de la información publicada por la Guía de Posgrados de la República Argentina, 2002. Véase Anexo 2. 


\section{LOS POSGRADOS DE LA UNIVERSIDAD DE BUENOS AIRES (UBA)}

La oferta que aparece en la Guía de Estudios de Posgrado de la UBA 2002 permite identificar algunas tendencias compatibles con las descritas para la Argentina y la región. La UBA ofrece 223 cursos de posgrado. De ellos, el 66,4 por ciento corresponde a las carreras de especialización, el 27,8 por ciento, a maestrías, y el 5,8 por ciento, a los doctorados. Entre las carreras de especialización, Medicina ofrece el 39 por ciento del total y le siguen Arquitectura y Ciencias Económicas, con el nueve por ciento de las carreras de especialización y Farmacia y Bioquímica, Derecho e Ingeniería, cada una con el ocho por ciento de las especializaciones. En materia de maestrías, la Facultad de Agronomía ofrece catorce programas; Filosofía y Letras, once; Ingeniería, nueve, y Ciencias Exactas y Naturales ofrece ocho maestrías. Los programas de maestría ofrecidos por estas cuatro facultades representan el 66 por ciento del total de maestrías. Desde 2001 hasta el momento de la publicación de la Guía en el 2002, se crearon trece nuevas carreras de especialización y ocho nuevas maestrías. El mercado de trabajo, las corporaciones y algunos grupos de académicos configuran la fuerza que impulsa gran parte de estas tendencias desde mediados de la década de 1990, cuando se produjo una explosión de carreras de especialización y maestrías. Se buscó capacitar y actualizar profesionales, así como ampliar los recursos financieros de las distintas unidades académicas ya que los posgrados son arancelados.

En el Gráfico 1 se registran los inscritos en los posgrados de la Universidad de Buenos Aires desde 1990 hasta 2002, según los sectores institucionales.

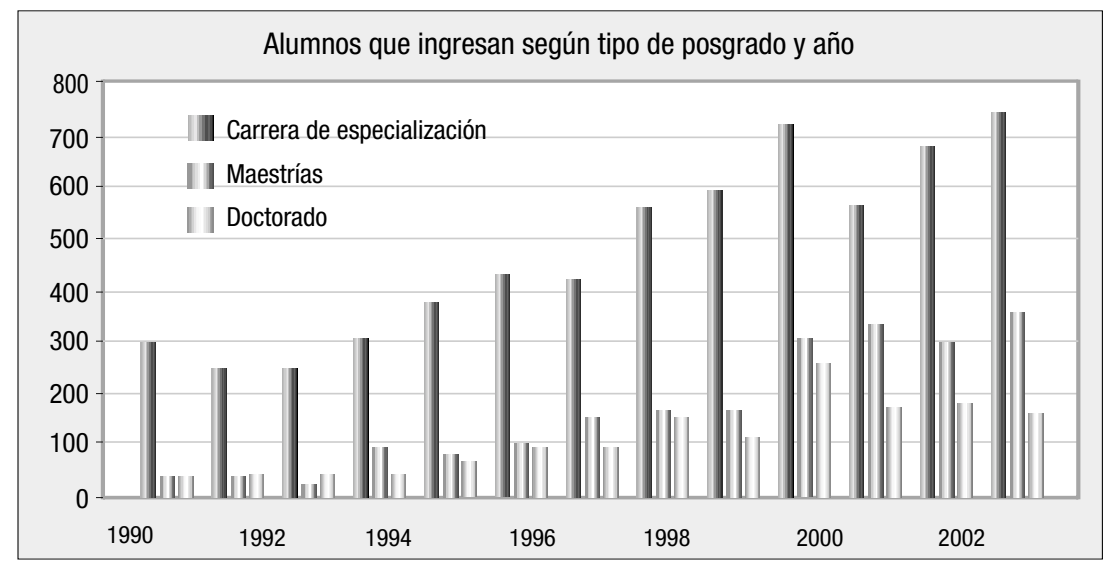

Fuente: Anexo 1, cuadros 1, 2 y 3. 
El Gráfico 1 permite comprobar el incremento constante del número de alumnos que ingresan a los posgrados de la UBA, producido desde 1990 hasta el 2002. Las especializaciones alcanzan su mayor número de inscritos en el 2002, en tanto que los inscritos al doctorado lo hacen en 1999. Ese número ha descendido desde entonces hasta el 2002. La matrícula de las especializaciones en 1990 representaba un ochenta por ciento del total, la de las maestrías un 9,5 por ciento y los inscritos al doctorado, representaban el 10,5 por ciento. En cambio, en el 2002 las especializaciones representan el sesenta por ciento del total, las maestrías el veintiocho por ciento de la matrícula y los doctorados, el doce por ciento. Si bien estos porcentajes no reflejan el total de inscritos en los posgrados de la UBA, ya que no representan a la totalidad de las unidades académicas, resulta evidente la supremacía desarrollada por las maestrías con respecto al doctorado, a partir de la segunda mitad de la década de 1990.

\section{Deserción, permanencia y egreso de los posgrados de la UBA}

Dado que se carece de suficientes investigaciones que analicen las causas de la deserción en los posgrados argentinos, nos referiremos a las condiciones que facilitan o dificultan que ellos sean completados.

Existen dos tipos de condiciones: las correspondientes a quien cursa el posgrado y las de los programas ofrecidos. Respecto a las primeras, es posible identificar dos tipos. Por un lado, las condiciones para escribir y defender una tesis de maestría o doctorado, que son prácticamente las mismas que requiere la práctica de la investigación. Sin embargo, la mayoría de los aspirantes a los posgrados no tiene el perfil del investigador.

A la vez, las diferentes formaciones adquiridas durante los estudios de grado influencian al aspirante al posgrado en su desempeño futuro. Algunas carreras incorporan en su plan de estudios actividades de investigación, becas de investigación para estudiantes o cuentan con un cuerpo de docentes/investigadores que promueven estrategias y habilidades vinculadas con la producción de conocimiento en los cursos de grado. Cuando ambas condiciones están ausentes, hay mayores probabilidades de abandonar el posgrado sin realizar la tesis. 
En cuanto a las condiciones de los posgrados, se reconocen tres tipos de programas que, desde una perspectiva histórica, tienen una trayectoria diferente en la Argentina: los programas de doctorado, las maestrías y las carreras de especialización (Barsky 1994; Krotsch 1996)

El doctorado, desde la década de 1960 en adelante, representa el único programa de posgrado que cuenta con una tradición orientada hacia el perfil científico y la formación de investigadores o de profesionales con alto nivel de especialización. En la UBA existen carreras que ofrecen el doctorado en ciencias aplicadas y, por lo tanto, cuentan con un número importante de doctores que no desarrollan su profesión en el campo científico, por ejemplo, Agronomía, Geología, Bioquímica y Farmacia, entre otras. En estos casos, la formación en la investigación aplicada se brinda desde la oferta curricular de grado, garantizando mayores probabilidades de éxito a los candidatos al doctorado en dichas disciplinas.

Las maestrías en la UBA surgen a mediados de la década de 1980, aunque su explosión junto con las carreras de especialización se produjo a mediados de 1990. Más de la mitad de los posgrados han sido creados a partir de entonces como respuesta a la regulación que introdujo la Ley de Educación Superior, promulgada en 1995; a la dinámica interna de las propias unidades académicas y, en menor medida, como respuesta a un tipo de demanda selectiva del mercado de trabajo de las grandes corporaciones.

A partir de la información relevada recientemente por la Subsecretaría de Posgrado $^{5}$ de la UBA, resulta posible analizar un conjunto de condiciones que están presentes en los programas de las maestrías. Cuando se orientan a la capacitación profesional de los adultos insertos en el mercado de trabajo y tienen un plan de estudios organizado en torno a cursos aislados, es muy probable que los candidatos abandonen el programa antes de terminar sus tesis. En cambio, los programas de maestría que favorecen el egreso con la defensa de tesis incluida, se caracterizan por:

5. Informe Preliminar sobre la Situación de los Posgrados en la UBA, Subsecretaría de Posgrado, Secretaría de Asuntos Académicos, UBA, mayo 2003 (versión mimeográfica). 
- la selección de candidatos por cohorte cerrada,

- el apoyo estratégico del empleador del aspirante, a través de licencias rentadas o becas que cubren los aranceles a través de convenios institucionales

- el compromiso del empleador con el candidato en cuanto a su reinserción laboral futura

- los talleres de tesis que acompañan la oferta curricular en los trayectos iniciales

- la presencia de investigadores en el plantel de profesores

- un plan de estudios que combina trayectos formativos obligatorios con circuitos optativos

Las carreras de especialización de la UBA suelen presentar altos índices de deserción. Los adultos que trabajan y perciben la necesidad de actualizarse asisten para satisfacer necesidades específicas de perfeccionamiento y luego los abandonan. En algunos casos, la relación entre las demandas del empleador y las exigencias de los cursos de especialización no coinciden y obligan al aspirante a desertar. En otros casos, las dificultades financieras del alumno provocan la deserción.

Dos razones significativas explican la explosión de la matrícula de los cursos de especialización en la UBA, durante la década de 1990. La primera, es la demanda de actualización por parte de los estudiantes, generada en un mercado de empleo más selectivo. La segunda, se refiere a los intereses particulares de grupos de personas que organizan su oferta para satisfacer la motivación credencialista, sin considerar la racionalidad académica para ayudar al aspirante a culminar la especialización con éxito.

En cualquiera de esos casos, existen condiciones del candidato y de los programas que pueden facilitar y favorecer los trayectos exitosos en los posgrados. Resulta, pues, indispensable promover programas académico-científicos, así como condiciones laborales y sociales que aumenten las posibilidades de egreso de los aspirantes, abandonando así la exclusiva finalidad de lucro que caracterizó la explosión de los posgrados en la década de 1990. 
UN BALANCE POSIBLE: AUSENCIA DE PRIORIDADES PARA LA PERTINENCIA SOCIAL Y FALTA DE PLANIFICACIÓN PARA EL DESARROLLO CIENTÍFICO

La moderna educación superior masiva enseña a la gente a no dedicarse casi por entero a una ocupación o a un determinado conjunto de aptitudes. La prepara para la eventualidad de que ambas cosas cambien a menudo y de que tengan que moverse con celeridad. Para moverse con celeridad hay que acarrear poco peso $0^{6}$.

En gran medida, los estudios sobre los posgrados destacan su débil vinculación con el mercado de trabajo. Por el contrario, demuestran que su expansión respondió a una demanda de mercados institucionales, en particular de las propias universidades, debido fundamentalmente al requisito del diploma de posgrado -maestría y/ o doctorado- para progresar en la carrera académica, y a la necesidad de búsqueda de financiamiento alternativo. El crecimiento desordenado y caótico de las especialidades reproduce la escena de un supermercado de conocimientos (Mollis 2003), dispuesto a satisfacer una clientela deseosa de actualización, más que de saberes continuos u habilidades y estrategias superiores que requieran esfuerzo intelectual, como lo describe Gibbons.

Por otro lado, en la mayoría de los casos la matrícula de los posgrados se concentra en áreas no consideradas prioritarias. En efecto, llama la atención, por ejemplo, la matrícula insuficiente en el área de la agroindustria cuando existen necesidades alimentarias tan graves no sólo en la Argentina sino en todos los países de la región.

Los estudiosos de este problema reconocen algunas tareas urgentes para lograr que los posgrados satisfagan los requerimientos de pertinencia social que promueven la Organización de Naciones Unidas para Educación, Ciencia y Cultura (UNESCO) y el Instituto Internacional para la Educación Superior de América Latina y el Caribe (IESALC), (2003). Algunas de esas tareas urgentes son:

- la necesidad de identificar las prioridades inmediatas y de largo plazo en disciplinas cruciales para el desarrollo científicotecnológico de nuestros países

6. M. Gibbons, p. 14. 
- promover desarrollos interdisciplinarios, mediante la fusión o articulación de carreras de especialización y/o maestrías, aprovechando los recursos disponibles en las diferentes unidades académicas

- identificación de líneas de formación más avanzadas, con ventajas comparativas en el contexto global

- estrechar relaciones con el campo regional e internacional, a través de convenios que promuevan intercambios entre docentes e investigadores

- promover la articulación entre los posgrados regionales del MERCOSUR y latinoamericanos, mediante acciones conjuntas en base al desarrollo de las disciplinas y grupo de pares

- mayor articulación de los posgrados con otras instituciones de la sociedad civil, por ejemplo, programas destinados a la prevención y fomento de la salud familiar, la transparencia cívica, el presupuesto del gobierno local, la tecnología de alimentos, la formación de formadores, la gestión, organización y sistematización de las instituciones educativas locales y regionales, etc.

En la medida que la excelencia científica se vincula con el desarrollo del conocimiento profesional y la investigación de nivel internacional, y necesita contar con el acuerdo de la comunidad académica, también se requiere planificar los programas a partir de prioridades que acerquen el conocimiento de los expertos a la ciudadanía. La planificación y el establecimiento de prioridades ayudarán a limitar la expansión caótica de los posgrados, en la actualidad orientados al servicio del cliente, y a fomentar, en cambio, los posgrados de excelencia cuyo objetivo sea el desarrollo de la cultura, las ciencias y la tecnología, en contextos regionales y locales que plantean necesidades sociales apremiantes. 


\section{Anexo 1}

Total de alumnos de las carreras de especialización, por año, según Unidad Académica en la UBA.

\begin{tabular}{|lcrrrrrrrrrrrrrr|}
\hline Unidad Académica & Año & & & & & & & & & & & & \\
& 1990 & 1991 & 1992 & 1993 & 1994 & 1995 & 1996 & 1997 & 1998 & 1999 & 2000 & 2001 & 2002 \\
\hline Agronomía & & & & & & & & 8 & 1 & 1 & 2 & 28 & 57 \\
\hline Arquitectura & 52 & 59 & 63 & 59 & 65 & 70 & 94 & 81 & 234 & 264 & 205 & 242 & 241 \\
\hline CEA & 37 & & 25 & 74 & 6 & 53 & 42 & 49 & 28 & 39 & 14 & 18 & 22 \\
\hline Cs. Exactas & 18 & 9 & 10 & 17 & 21 & 28 & 39 & 37 & 35 & 33 & 39 & 52 & 51 \\
\hline Cs. Sociales & & & & 39 & & 40 & & 31 & 39 & & 29 & & 44 \\
Cs. Veterinarias & 58 & 65 & 33 & 47 & 50 & 49 & 35 & 30 & 53 & 122 & 120 & 58 & 77 \\
Farmacia y Bioq. & & & & & 127 & 55 & 41 & 114 & 37 & 95 & 30 & 89 & 45 \\
\hline Ingeniería & 82 & 97 & 101 & 38 & 93 & 114 & 146 & 132 & 134 & 61 & 98 & 67 & 168 \\
\hline Odontología & 11 & 15 & 6 & 3 & 15 & 18 & 24 & 18 & 29 & 28 & 26 & 46 & 27 \\
\hline Psicología & 40 & & 8 & 30 & & 3 & & 61 & & 75 & & 74 & 16 \\
\hline Total general & 298 & 245 & 246 & 307 & 377 & 430 & 421 & 561 & 590 & 718 & 563 & 674 & 748 \\
\hline
\end{tabular}

Total de estudiantes de maestrías, por año, según Unidad Académica en la UBA.

\begin{tabular}{|lcrrrrrrrrrrrr|}
\hline Unidad Académica & Año & & & & & & & & & & & \\
& 1990 & 1991 & 1992 & 1993 & 1994 & 1995 & 1996 & 1997 & 1998 & 1999 & 2000 & 2001 & 2002 \\
\hline Agronomía & 16 & 22 & 4 & 34 & 27 & 32 & 18 & 35 & 13 & 66 & 62 & 69 & 56 \\
Arquitectura & & & & & & 1 & 3 & 1 & 12 & 20 & 22 & 14 & 13 \\
CEA & 20 & & 18 & 46 & 39 & 27 & 41 & 77 & 38 & 45 & 64 & 38 & 65 \\
Cs. Exactas & & 7 & & 2 & & 17 & 3 & & 1 & 63 & 43 & 71 & 72 \\
\hline Cs. Sociales & & & & & & 23 & 22 & & 40 & 22 & 60 & 24 & 64 \\
Cs. Veterinarias & & 10 & & 9 & 5 & & & 14 & & 8 & & & \\
Farmacia y Bioq. & & & & & 9 & & 20 & & 16 & & 14 & & 13 \\
\hline Ingeniería & & & & & & & 6 & 9 & 15 & 6 & 13 & 22 & \\
Odontología & & & & & & & & 3 & 6 & 5 & & & 6 \\
\hline Psicología & & & & & & & & & & 69 & 20 & 38 & 40 \\
\hline Salud Pública & & & & & & & 40 & 30 & 25 & & 36 & 22 & 23 \\
\hline Total general & 36 & 39 & 22 & 91 & 80 & 100 & 153 & 169 & 166 & 304 & 334 & 298 & 352 \\
\hline
\end{tabular}

Fuente: Base de datos de información, recogida por la Subsecretaría de Posgrado de la Secretaría de Asuntos Académicos de la UBA, programación 2003.

NOTA La información de las facultades de Ciencias Económicas, Derecho y Filosofía y Letras no permite discriminar entre alumnos inscritos en cursos y los admitidos en las carreras de especialización o maestría, por esta razón no fue procesada. La Facultad de Medicina, al momento de la publicación, no había enviado antecedentes: 
Total de estudiantes de doctorados, por año, según Unidad Académica en la UBA.

\begin{tabular}{|c|c|c|c|c|c|c|c|c|c|c|c|c|c|}
\hline \multirow[t]{2}{*}{ Unidad Académica } & \multicolumn{13}{|l|}{ Año } \\
\hline & 1990 & 1991 & 1992 & 1993 & 1994 & 1995 & 1996 & 1997 & 1998 & 1999 & 2000 & $0 \quad 2001$ & 2002 \\
\hline Agronomía & & & & & & & 5 & & 3 & 8 & 13 & 310 & 20 \\
\hline \multicolumn{14}{|l|}{ Arquitectura } \\
\hline \multicolumn{14}{|l|}{ Cs. Exactas } \\
\hline Cs. Sociales & & & & & & & & & & 62 & 41 & 34 & 33 \\
\hline Cs. Veterinarias & 10 & 3 & 7 & 8 & 13 & 14 & & 36 & & 27 & 16 & 12 & 1 \\
\hline Farmacia y Bioq. & 25 & 35 & 35 & 29 & 46 & 58 & 45 & 71 & 59 & 70 & 55 & 63 & 70 \\
\hline Ingeniería & & & & 1 & 1 & 4 & 19 & 8 & 9 & 12 & 8 & 18 & 8 \\
\hline Odontología & 5 & 5 & 2 & 5 & 4 & 4 & 6 & 3 & 7 & 9 & 11 & 18 & 5 \\
\hline Psicología & & & & & & 9 & 18 & 33 & 36 & 70 & 26 & 23 & 22 \\
\hline Total general & 40 & 43 & 44 & 43 & 64 & 89 & 93 & 151 & 114 & 258 & 3170 & 178 & 3159 \\
\hline
\end{tabular}




\section{Anexo 2}

Listado actualizado de las carreras de posgrado acreditadas por la CONEAU en universidades públicas y privadas argentinas.

\begin{tabular}{|c|c|c|c|c|}
\hline & & CONEAU & & \\
\hline & Especializaciones & Maestrías & Doctorados & Total \\
\hline Administración & 32 & 33 & 3 & 68 \\
\hline Agronomía & 7 & 32 & 4 & 43 \\
\hline Antropología & - & 1 & 1 & 2 \\
\hline Arquitectura & 9 & 9 & 1 & 19 \\
\hline Artes & - & 3 & 1 & 4 \\
\hline Astronomía & - & - & 2 & 2 \\
\hline Biología & 1 & - & - & 1 \\
\hline Bioquímica & 18 & 6 & 4 & 28 \\
\hline Ciencias Biológicas & - & 7 & 11 & 18 \\
\hline Ciencias de la Educación & 16 & 26 & 1 & 43 \\
\hline Ciencias de la Tierra & - & 4 & 9 & 13 \\
\hline Ciencias Jurídicas & 46 & 10 & 10 & 66 \\
\hline Ciencias Naturales & - & 1 & 1 & 2 \\
\hline Ciencias Políticas & - & 4 & 1 & 5 \\
\hline Ciencias Sociales & 6 & 15 & - & 21 \\
\hline Comunicación & - & 3 & 1 & 4 \\
\hline Defensa y Seguridad & - & 5 & - & 5 \\
\hline Demografía & - & 2 & - & 2 \\
\hline Economía & 2 & 12 & 5 & 19 \\
\hline Farmacia & 4 & - & 1 & 5 \\
\hline Filosofía & 1 & 6 & 7 & 14 \\
\hline Física & - & 2 & 11 & 13 \\
\hline Geografía & - & - & 3 & 3 \\
\hline Historia & 1 & 5 & 7 & 13 \\
\hline Informática & 1 & 9 & 5 & 15 \\
\hline Ingeniería & 21 & 38 & 24 & 83 \\
\hline Letras & 1 & 11 & 4 & 16 \\
\hline Matemática & - & 7 & 7 & 14 \\
\hline Medicina & 237 & 23 & 8 & 268 \\
\hline Medio Ambiente & 6 & 7 & - & 13 \\
\hline Meteorología & 6 & 7 & - & 13 \\
\hline Odontología & 10 & 1 & 3 & 14 \\
\hline Políticas Sociales & 3 & 4 & - & 7 \\
\hline Psicología & 8 & 10 & 5 & 23 \\
\hline Química & 1 & 7 & 12 & 20 \\
\hline Relaciones Internacionales & 2 & 11 & 1 & 14 \\
\hline Tecnología de Alimentos & & 8 & 7 & 15 \\
\hline Veterinaria & 2 & 3 & 3 & 8 \\
\hline TOTAL & 441 & 332 & 163 & 936 \\
\hline
\end{tabular}

Fuente: Guía de Posgrados de la República Argentina 2002, Lázara Grupo Editor, Buenos Aires. 


\section{REFERENCIAS BibLIOGRÁFICAS y NOTAS}

Brünner, José Joaquín (1990). Educación Superior en América Latina. Cambios y Desafíos, Fondo de Cultura Económica, Chile

Barsky, Osvaldo (1994). Análisis del sistema argentino de ofertas de posgrado, Secretaría de Políticas Universitarias, Ministerio de Educación y Cultura, Argentina.

Guía de Estudios de Posgrado 2002. Subsecretaría de Posgrado de la UBA, EUDEBA, Buenos Aires.

Guía de Posgrados de la República Argentina 2002. Lázara Grupo Editor, Buenos Aires.

García Guadilla, Carmen (1994). "Posgrados en América Latina: elementos de análisis", en Pensamiento Universitario, Año 2, $\mathrm{N}^{\circ} 2$, Buenos Aires.

García Guadilla, Carmen (1996). Situación y Principales Dinámicas de Transformación de la Educación Superior en América Latina, Ediciones CRESALC/UNESCO, Caracas.

Gibbons, Michael (1998). "Pertinencia de la educación superior en el siglo XXI", Contribución a la Conferencia Mundial sobre Educación Superior de la UNESCO, UNESCO, París.

IESALC/UNESCO (2003), boletín@unesco.org.ve

Krotsch, Pedro (1996). "El posgrado en la Argentina: una historia de discontinuidad y fragmentación", en Pensamiento Universitario, Oficina de Publicaciones CBC, $N^{\circ} 4 / 5$, Buenos Aires.

Mollis, Marcela (coord.) (2003). Universidades en América Latina: ¿Reformadas o Alteradas?, Editorial CLACSO, Buenos Aires.

Mollis, Marcela (1996). "La evaluación de la calidad universitaria en la Argentina", en: Costa Morosini, Marilia (org) Universidade No Mercosul, Cortez Editora, Sâo Paulo, Brasil. 
RICYT (Red de Indicadores de Ciencia y Tecnología Iberoamericana / Interamericana) 2000. El Estado de la Ciencia. Principales Indicadores de Ciencia y Tecnología, Buenos Aires.

Subsecretaría de Posgrado de la UBA (2003). Informe Preliminar sobre Situación de los posgrados de la UBA, Buenos Aires (versión mimeográfica). 